\title{
Recovery of Indium form Waste LCD by Solvent Extraction with Ionic Liquids
}

\author{
Xiaofei Yin, ${ }^{1}$ Kaihua Zhang, ${ }^{1}$ and Yufeng Wu, ${ }^{1}{ }^{*}$ \\ ${ }^{1}$ Institute of Circular Economy, Beijing University of Technology, Beijing, 100124, P. R. China. \\ * Corresponding author: E-mail:yxfxunhuan@163.com; Street address: No. 100, PingleyuanStreet, Chaoyang \\ District, Beijing, 100124, China; Tel: +86-10-67396263; Fax: +86-10-67396263.
}

\begin{abstract}
A green solvent extraction process for recycling indium from waste LCD has been developed employing ionic liquids. Methyl trioctyl ammonium chloride [A336][Cl] was identified as the most suitable extraction agent due to synergistic effect. The effects of various parameters such as extraction time, acidity and O/A phase ratio on the extraction process were explored. Experiments results showed that the optimal conditions for extraction of In from the waste LCD leaching solution were O/A phase ratio of 1:5, the HCl concentration of $4 \mathrm{M}$, and reaction time of $3 \mathrm{~min}$, thus the distribution ratio reached 400 . The extracted oil phase is stripped by oxalic acid $(3 \mathrm{~mol} / \mathrm{L})$, the $\mathrm{Fe}$ and Sn would transfer into the liquid phase, while $\mathrm{InCl}_{n}^{-}$keeps bonding with Aliquat336. While scrubbing with aqua pura, the one-time stripping efficiency of In reaches $92.63 \%$, and the ionic liquid can be purified and recycled through the stripping and scrubbing.
\end{abstract}

Keywords: waste LCD, recycling, indium, ionic liquid.

\section{Introduction}

Indium (In) is known as a scarce metal because of its extremely low concentration in the crust of earth, which has been reserved as a strategic resource by the United States, Japan and other countries.[1] Based on its excellent conductivity and optical transparency, indium has been widely used as functional materials in many fields such as Liquid crystal display (LCD), electronic semiconductor, solder alloy and so on.[2] Statistics shows that almost $75 \%$ of the indium production are made into indium tin oxide film (ITO) $\left(\operatorname{In}_{2} \mathrm{O}_{3}: \mathrm{SnO}_{2}=9: 1\right)$ and used in LCD and solar thin-film battery.[3] With the continue increase of consumer demand, the reserve of indium is rapidly decreased and the sustainable application of indium meets bottlenecks. The recycling of secondary indium resources has been an effective approach to mitigate the exhaustion of the primary minerals of indium. With the rapid development of modern display industry, ITO screen has been widely used in LCD TV, flatscreen televisions, smart phones and other electronic products.[4] On the other hand, a large number of LCD were discarded and stockpiled as hazardous materials due to the fact that the LCD products have a relatively short service life (about 3-5 years).[5] The recycling of indium from waste LCDs has been a hot topic, which is expected to efficiently ease the tight application of indium resource, and has a considerable development prospect.

In the existing recycling research studies, hydrometallurgy involving leaching and extraction has been the most common method used. Many researches have focused on the leaching of indium from waste LCDs, however, researches on the separation and purification of indium from the lixivium are still lack. In the actual production, the requirement for the purity of indium is very high. Thus, the purification after leaching is necessary when recycling indium from waste LCDs. Yang et al. investigated the extraction of In from waste LCD lixivium using P204. Results showed that through controlling the extraction time, the main impurity Fe can be removed efficiently. In the optimal condition of acidity $0.5-1.5 \mathrm{~mol} / \mathrm{L}, \mathrm{O} / \mathrm{A} 1: 5$ and centrifugal extraction for 3-5 min, the extraction rate of In can reach 100\%. Ruan et al.[6] explored the effects of $\mathrm{D}_{2} \mathrm{EHPA}$ and aviation kerosene on the extraction of In. Results showed that under the conditions of O/A =1:5, 30\% $\mathrm{D}_{2} \mathrm{EHPA}$ and $70 \%$ aviation kerosene as diluent for $5 \mathrm{~min}$, the extraction rate of In can reach 92\%. Inoue et al.[7] developed an efficient recovery process for In from waste liquid crystal display panels using porous resins impregnated with 
each of Cyanex 923 and Aliquat 336. The extracted metals can be mutually separated and recovered by passing through the column packed with the resin impregnated with Aliquat 336 ( $\mathrm{Sn}, \mathrm{Fe}$ and $\mathrm{Zn}$ ) followed by that with Cyanex 923 (Zn). However, the existing methods are still using traditional organic extractant, which are volatile and a potential threat to the environment. And the emerging liquid membrane and resin separation technology often suffer from e low extraction separation efficiency.[8, 9] Therefore, the key point for recycling indium form LCD is to choose the environmental friendly and high efficiency extraction solvent for separation and purification. Ionic liquid (IL)is a kind of burgeoning green alternative solvent to conventional VOSs [10] due to the excellent characteristics such as high heat capacity, low vapor pressure and stability of their properties. [11]

In this paper, IL was employed to extract In from the pickling liquid of waste LCD. First, a high-efficiency, low-cost, and pollution-free extraction agent was selected. In addition, the effects of variables such as extraction time, organic phase / inorganic phase and acid concentration on the extraction efficiency were investigated. Moreover, the optimal reverse extraction agent and conditions were explored, and the ionic liquid can be purified and recycled through the stripping and scrubbing.

\section{Experimental Section}

\subsection{Materials and Regents}

All reagents used in this study were of the highest available purity, purchased from Chemical Reagent Company of Beijing. The IL used in this study was supplied by Shanghai chengjie chemical co., Ltd (Shanghai, China). The extraction agent were purchased from Luoyang Aoda Chemical Co., Ltd (Luoyang, China). Deionized water (18.2 M $\Omega . c m$ ) obtained from a Direct-Pure Up 10 water system (RephiLe Bioscience, Ltd.) was used through the experiments whenever needed. In and other elements in waste LCD were leached successfully in our former research. And the main contents of the lixivium was $\operatorname{InCl}_{\mathrm{n}}{ }^{-}(283 \mathrm{mg} / \mathrm{L}), \mathrm{Al}^{3+}(156.2 \mathrm{mg} / \mathrm{L}), \mathrm{Mg}^{2+}$ $(31.8 \mathrm{mg} / \mathrm{L}), \mathrm{Ca}^{2+}(20.2 \mathrm{mg} / \mathrm{L}), \mathrm{Fe}^{3+}(30 \mathrm{mg} / \mathrm{L}), \mathrm{Sn}^{2+}(17.5 \mathrm{mg} / \mathrm{L}), \mathrm{Na}^{+}(4 \mathrm{mg} / \mathrm{L})$, and the acidity was $0.8 \mathrm{M} \mathrm{HCl}$.

\subsection{Analysis equipment}

HJ-6A multiposition magnetic stirrer hotplate (Guohua) applied to achieve mixing uniformly in the process of extraction. The PH measurements carried out by a PB-10 digital PH-meter (Sartorius, German). Optima 8000 inductively coupled plasma-optical emission spectrometer (ICP-AES) (PerkinElmer, USA) was performed to determine the concentration of metals in the aqueous phase before and after extraction. All extraction experiments performed by three times to ensure the reproducibility of the values, and relative standard deviations were found to be within $\pm 1 \%$.

\subsection{Methodology}

Firstly, to determine which kind of extractant and ionic liquid is efficient to extraction In from waste LCD acid leaching solution, different organic extractants and ionic liquids were extracted selectively. All waste ITO glasses were leached by $0.8 \mathrm{~mol} / \mathrm{L} \mathrm{HCl}(\mathrm{aq})$ in this experiment and then extracted with $1: 10 \mathrm{O} / \mathrm{A}$ for $30 \mathrm{~min}$ by aforementioned extraction agents at room temperature. After the extraction process reached equilibrium, the ion concentration of In in the aqueous phase were measured by ICP-AES with triplicates. After extractant and IL were determined, a series of experiments about the effect of acid concentration in leaching liquor, phase ratio, extraction time was investaged. The extraction percentage (E\%) and distribution ratio (D) could be written using the following equations:

$$
\begin{gathered}
D=\frac{\left([M]_{0}-[M]_{a q}\right) V_{a q}}{[M]_{a q} V_{\text {org }}} \\
E \%=\frac{V_{\text {org }}\left([M]_{0}-[M]_{a q}\right)}{[M]_{0} V_{a q}} \times 100 \%
\end{gathered}
$$


Where $[\mathrm{M}]_{0}$ and $[\mathrm{M}]_{\mathrm{aq}}(\mathrm{mol} / \mathrm{L})$ represents the metal concentration in the aqueous phase before and after extraction, respectively. $\mathrm{V}_{\text {aq }}$ and $\mathrm{V}_{\text {org }}(\mathrm{mL})$ are the volumes of the aqueous phase and ionic liquid phase, respectively.

\section{Results and Discussion}

\subsection{Selection of Appropriate Extraction Systems}

For the purpose of comparison, the different extraction systems, BmimPF $6, \mathrm{BmimNTF}_{2}$, Joint extraction agents $\left(\mathrm{P} 204+\mathrm{BmimPF}_{6}, \mathrm{P} 204+\mathrm{BmimNF}_{2}, \mathrm{Cyanex}\right.$ 23+BmimPF $6, \mathrm{Cyanex}$ 23+BmimNTF $2, \mathrm{PC} 88 \mathrm{~A}+\mathrm{BmimPF}$, $\mathrm{PC} 88 \mathrm{~A}+\mathrm{BmimNTF} 2), \mathrm{A} 336 \mathrm{NO}_{3}, \mathrm{~A} 336 \mathrm{NO}_{3}+\mathrm{Cyanex} 923, \mathrm{~A} 336 \mathrm{Cl}$, and $\mathrm{A} 336 \mathrm{Cl}+\mathrm{Cyanex} 923$, were designed to extract the major elements from waste LCD. Then extraction rates of In were showed in Fig. 1(a). It's obvious to find that single $\mathrm{BmimPF}_{6}$, or $\mathrm{BmimNTF}_{2}$ was not suitable for extracting In with low extraction rate. This is because negative ions in $\mathrm{BmimPF}_{6}$ and $\mathrm{BmimNTF}_{2}$ are lack of special functional group. So, in this experiment, the joint extraction agents including imidazolium-based ionic liquids and traditional extraction agents, were used to fix this flaw and then acted better with 30\%-50\% extraction rate than single imidazolium-based ionic liquid. Though the joint extraction was confirmed available, functional quaternary ammonium-based ionic liquids achieved a much satisfactory work. The extraction rates of single $\mathrm{A} 336 \mathrm{NO}_{3}, \mathrm{~A} 336 \mathrm{Cl}$ were almost $80 \%$ and the extraction rates of $\mathrm{A} 336 \mathrm{NO} 3+$ Cyanex923, A336Cl+ Cyanex923 even exceeded 90\%. Comparing these extraction systems, the quaternary ammonium-based ionic liquids was better choice to extract In.

To make sure the best extraction system, the extraction rates of main elements extracted by $\mathrm{A}_{336 \mathrm{NO}_{3} \text { and }}$ $\mathrm{A} 336 \mathrm{Cl}$ in the ITO glasses leaching liquids, were compared in the Fig. 1(b). It indicated that the extraction

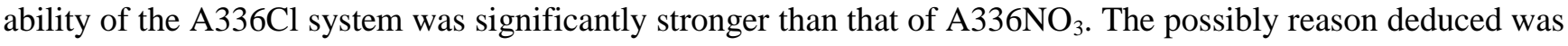
because the stronger electronegativity of $\mathrm{Cl}^{-}$could build closer bond with metal ions. [12] In this experiment, A336Cl system was chose as extraction agent.

(a)

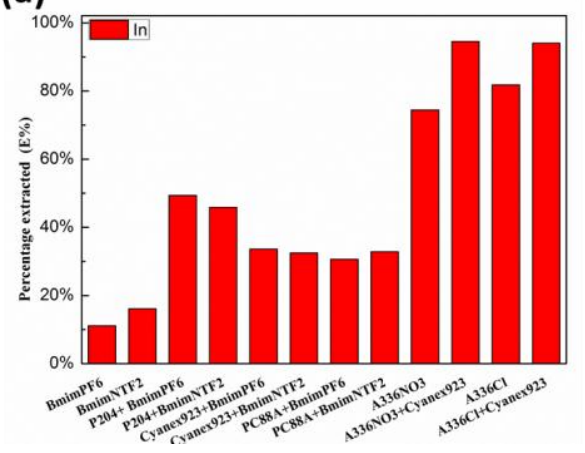

(b)

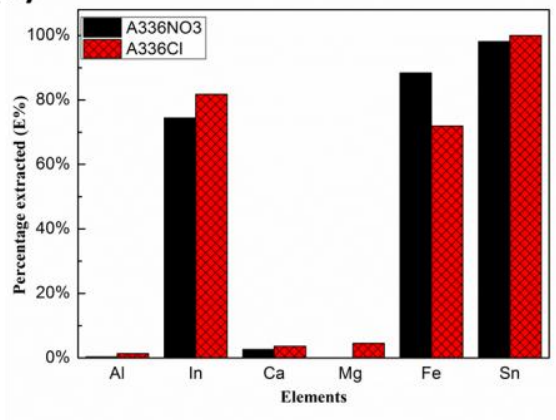

Fig. 1: (a) The extraction rates of In by various extracted agents, (b) The extraction rates of main elements by quaternaryammonium ionic liquids.

\subsection{Effect of Extraction Time}

To obtain the best experiment parameters, the effect of extraction time on indium extraction was researched. Taking $\mathrm{A} 336 \mathrm{Cl}$ as the extraction agent, $\mathrm{O} / \mathrm{A}=1: 10$, waste $\mathrm{ITO}$ glasses leaching agent of $0.8 \mathrm{~mol} / \mathrm{L} \mathrm{HCl}$ (aq) was made use of extract In for leaching times that varied from $3 \mathrm{~min}$ to $30 \mathrm{~min}$. And the result was shown in Fig. 2 below. On the whole, the In extraction rate increased from $83.13 \%$ to $87.42 \%$ with the time increased from $3 \mathrm{~min}$ to $30 \mathrm{~min}$ and the reaction was balanced at about $5 \mathrm{~min}$. Besides, the Fe extraction was in equilibrium with only 10 min while Sn was with 30min. And the extraction rate of Fe, Sn could reach $90 \%, 94 \%$ respectively. So, in order to reduce the content of impurity elements ( $\mathrm{Fe}$ and $\mathrm{Sn}$ ), the extractive reaction would be required to finish at 3 min. As a result, In accomplish a high extraction rate of $83.13 \%$ and the experiment controlled the extraction rate of $\mathrm{Fe}, \mathrm{Sn}$ at $84.65 \%, 58.76 \%$ to make sure high purity extraction of indium. 


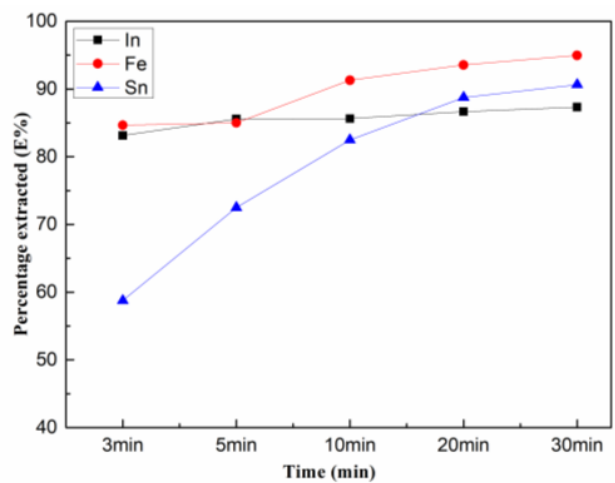

Fig. 2: The effect of time on main elements extraction in waste LCD leaching agent by $\mathrm{A} 336 \mathrm{Cl}$

\subsection{Effect of $\mathrm{O} / \mathrm{A}$}

Taking $\mathrm{A} 336 \mathrm{Cl}$ as the extraction agent, the experiment carry out the effects of $\mathrm{O} / \mathrm{A}$ on the extraction by varying the initial $\mathrm{O} / \mathrm{A}$ rate from $1: 1$ to $1: 20$ in solution containing $0.8 \mathrm{~mol} / \mathrm{L}$ waste $\mathrm{ITO}$ glasses leaching liquid for $3 \mathrm{~min}$ at room temperature. As shown in Fig. 3, when O/A was 1:1, the extraction rates of indium, iron and tin were all more than $95 \%$. With the decrease of O/A from 1:1 to 1:5, the extraction rate of each element decreased because of the reduced contact probability of metal ions and ionic liquids in solution. However, the extraction rate of indium remained high at over $90 \%$. It also depicted that the extraction rate of every element had been dropping with the O/A further decreasing. So, in order to reduce the consumption of ionic liquids and get better extraction rate, 1:5 O/A is preferred. As a result, the extraction rates of In, Sn, Fe were 92.11\%, 85.63\%, and $89.5 \%$, respectively.

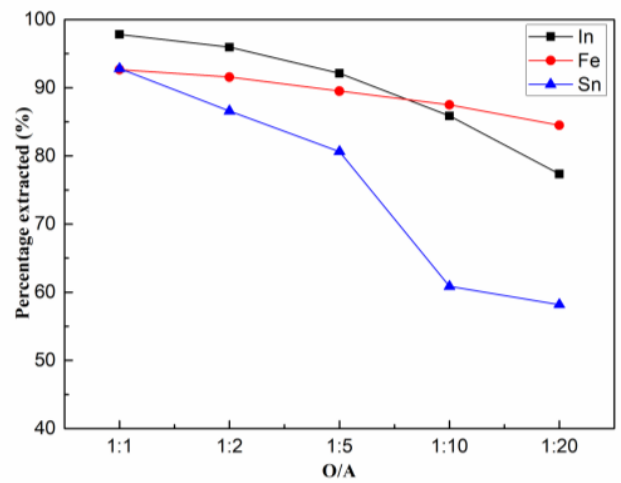

Fig. 3: The effect of O/A on main elements extraction in waste LCD leaching agent by $\mathrm{A} 336 \mathrm{Cl}$

\subsection{Effect of Acidity}

To obtain the best experiment parameters, the effect of acidity on indium extraction was researched. The experiments were performed by varying the acid concentration in the range of $0.1 \mathrm{~mol} / \mathrm{L}-10.0 \mathrm{~mol} / \mathrm{L}$ at room temperature with $\mathrm{O} / \mathrm{A}=1: 5$ for $10 \mathrm{~min}$. The extraction rate curve was shaped like a bell jar as shown in Fig. 4. Within the range 0 to $4 \mathrm{~mol} / \mathrm{L}$, the extraction rate went up with the acidity and reached its peak $98 \%$ at $4 \mathrm{~mol} / \mathrm{L}$. But when the acidity changed from $4 \mathrm{~mol} / \mathrm{L}-10 \mathrm{~mol} / \mathrm{L}$, the extraction distribution decreased with the increase of acidity. The addition of acid inhibited the extraction which was similar to the extraction of other metals by quaternary ammonium-based ionic liquid. Based on the research, $4 \mathrm{~mol} / \mathrm{L}$ was the optimum extraction acidity. 


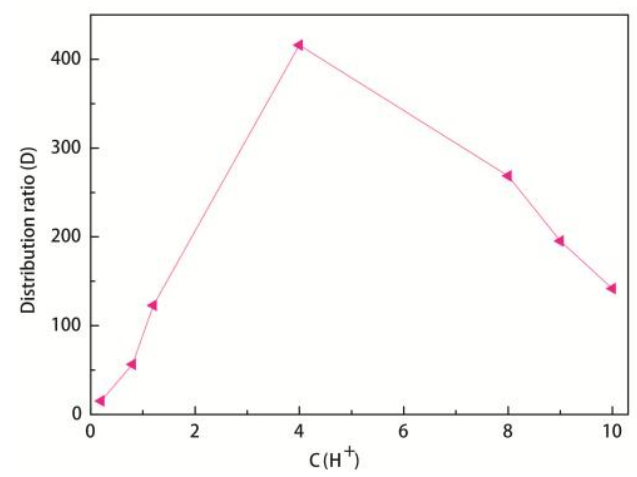

Fig. 4: The effect of acid concentration on indium extraction by $\mathrm{A} 336 \mathrm{Cl}$

\subsection{Stripping}

Oxalic acid was used for stripping to separate In from impurities. Table 1 shows the scrubbing effect of oxalic acid on $\mathrm{A} 336 \mathrm{Cl}$ extracted oil phase. After 3 times of scrubbing using $3 \mathrm{M}$ oxalic acid, almost $98 \%$ of the impurities such as $\mathrm{Al}, \mathrm{Mg}, \mathrm{Na}, \mathrm{Fe}$ and $\mathrm{Sn}$ can be stripped into aqueous phase, and In still stay in the IL phase. After that, the In ions can be furtherly scrubbing out employing aqua pura, and the one-time stripping rate can reach $94.14 \%$. On the other hand, the IL phase after two step stripping by oxalic acid and aqua pura was recovered. Figure 7 shows the contrast of extraction efficiency between recycled and original ionic liquid, which illustrates that the extraction ability of the recycled ionic liquid is not reduced and it can be reused for extraction.

TABLE 1: The scrubbing effect of oxalic acid on A336Cl extracted oil phase

\begin{tabular}{llll}
\hline \hline Elements & First & Second & Third \\
\hline $\mathrm{Al}$ & $0.23 \%$ & $72.42 \%$ & $22.08 \%$ \\
$\mathrm{In}$ & $0.23 \%$ & $0.18 \%$ & $1.19 \%$ \\
$\mathrm{Mg}$ & $14.84 \%$ & $29.22 \%$ & $36.72 \%$ \\
$\mathrm{Na}$ & $30.19 \%$ & $50.59 \%$ & $27.26 \%$ \\
$\mathrm{Fe}$ & $25.52 \%$ & $31.72 \%$ & $37.24 \%$ \\
$\mathrm{Sn}$ & $0.00 \%$ & $92.86 \%$ & $3.68 \%$ \\
\hline \hline
\end{tabular}

\section{Conclusion}

An efficient and green process for the extraction of indium from waste LCD lixivium was proposed in this work, and the following conclusions can be summarized.

(1) After comparation, $\mathrm{A} 336 \mathrm{Cl}$ was proved to be the most efficient and appropriate extraction agent for the extraction of In from waste LCD lixivium.

(2) Experiments results showed that the optimal conditions for extraction of In from the waste LCD leaching solution were $\mathrm{O} / \mathrm{A}$ phase ratio of 1:5, the $\mathrm{HCl}$ concentration of $4 \mathrm{M}$, and reaction time of $3 \mathrm{~min}$, thus the distribution ratio reached 400 .

(3) Oxalic acid (3mol/L) can be used for stripping In efficiently by two step scrubbing. Fe and Sn would transfer into the liquid phase by oxalic acid, and In can be furtherly scrubbed with aqua pura, the onetime stripping efficiency reaches $94.14 \%$. Moreover, the ionic liquid can be purified and recycled through the stripping and scrubbing.

\section{Acknowledgements}

This research was financially supported by Beijing Nova Program (Z1511000003150141), the Academician Workstation in Yunnan Province, and the Beijing Natural Science Foundation (2174067, 2174065). 


\section{References}

[1] A.M. Alfantazi and R.R. Moskalyk. "Processing of indium: a review," Minerals Engineering, vol.16, pp. 687-694, 2003.

[2] A. Yoshimura, I. Daigo, and Y. Matsuno. "Global Substance Flow Analysis of Indium," Materials Transactions, vol. 54, pp. 102-109, 2013.

[3] K. Zhang, Y. Wu, W. Wang, B. Li, Y. Zhang, and T. Zuo. "Recycling indium from waste LCDs: A review," Resources, Conservation and Recycling, vol. 104, pp. 276-290, 2015.

[4] B. Bessais, H. Ezzaouia, and R. Bennaceur. "Electrical Behavior and Optical-Properties of Screen-Printed ITO ThinFilms," Semiconducaor Science and Technology, vol. 8, pp. 1671-1678, 1993.

[5] H. Wang, Y. Gu, Y. Wu, Y. Zhang, and W. Wang. "An evaluation of the potential yield of indium recycled from endof-life LCDs: A case study in China," Waste Management, vol. 46, pp. 480-487, 2015.

[6] J. Ruan, Y. Guo, and Q. Qiao. Recovery of indium from scrap TFT-LCDs by solvent extraction. In: L. Jinhui, H. Hualong. (Eds.). Procedia Environmental Sciences, 2012, pp. 545-551.

[7] H. Hasegawa, I.M.M. Rahman, Y. Egawa, H. Sawai, Z.A. Begum, T. Maki, and S. Mizutani. "Recovery of indium from end-of-life liquid-crystal display panels using aminopolycarboxylate chelants with the aid of mechanochemical treatment," Microchemical Journal, vol.106, pp. 289-294, 2013.

[8] M. Erosa, T. Medina, R.N. Mendoza, M.A. Rodriguez, and E. Guibal. "Cadmium sorption on chitosan sorbents: kinetic and equilibrium studies," Hydrometallurgy. vol. 61, pp. 157-167, 2001.

[9] R. Biesuz, M. Pesavento, G. Alberti, F. and Dalla Riva. "Investigation on sorption equilibria of $\mathrm{Mn}(\mathrm{II}), \mathrm{Cu}(\mathrm{II})$ and Cd(II) on a carboxylic resin by the Gibbs-Donnan model," Talanta. vol. 55, pp. 541-550, 2001.

[10] X. Sun, B. Peng, Y. Ji, J. Chen, and D. Li. "The solid-liquid extraction of yttrium from rare earths by solvent (ionic liquid) impreganated resin coupled with complexing method," Separation \& Purification Technology, vol. 63, pp. 6168, 2008.

[11] Y. Baba, F. Kubota, N. Kamiya, and M. Goto. "Recent Advances in Extraction and Separation of Rare-Earth Metals Using Ionic Liquids," Journal of Chemical Engineering of Japan, vol. 44, pp. 679-685, 2011.

[12] M. Mohan Raj, A. Dharmaraja, K. Panchanatheswaran, K. A. Venkatesan, T. G. Srinivasan, and P. R. Vasudeva Rao. "Extraction of Fission palladium (II) From Nitric Acid by Benzoylmethylenetriphenylphosphorane (BMTPP)," Hydrometallurgy. vol. 84, pp. 118-124, 2006. 